Relations industrielles

Industrial Relations

\title{
Sept syndicalismes, par Gilles Martinet, Collection Histoire vivante, Paris, Seuil, 1979, 250 pp.
}

\section{Gérard Dion}

Volume 35, numéro 2, 1980

URI : https://id.erudit.org/iderudit/029070ar

DOI : https://doi.org/10.7202/029070ar

Aller au sommaire du numéro

Éditeur(s)

Département des relations industrielles de l'Université Laval

ISSN

0034-379X (imprimé)

1703-8138 (numérique)

Découvrir la revue

Citer ce compte rendu

Dion, G. (1980). Compte rendu de [Sept syndicalismes, par Gilles Martinet,

Collection Histoire vivante, Paris, Seuil, 1979, 250 pp.] Relations industrielles /

Industrial Relations, 35(2), 339-340. https://doi.org/10.7202/029070ar

Tous droits réservés (C Département des relations industrielles de l'Université Laval, 1980
Ce document est protégé par la loi sur le droit d'auteur. L'utilisation des services d'Érudit (y compris la reproduction) est assujettie à sa politique d'utilisation que vous pouvez consulter en ligne.

https://apropos.erudit.org/fr/usagers/politique-dutilisation/ 


\section{RECENSIONS BOOK REVIEWS}

Sept syndicalismes, par Gilles Martinet, Collection Histoire vivante, Paris, Seuil, 1979, 250 pp.

L'auteur se livre à une étude comparative des syndicalismes dans sept pays différents, comme il l'avait fait dans un ouvrage précédent à propos de Cinq communismes, (Paris, Seuil, 1971).

Dès le départ, il ne cache pas son identité. Les lecteurs savent à quelle enseigne il se loge et dans quel esprit il a entrepris son étude. «Je ne suis pas un observateur neutre; je suis un partisan du socialisme. Mais j'ai horreur des récits de complaisance, des romans édifiants et des dépliants de propagande. Je n'ai aucune envie de me faire plaisir en décrivant les faits tels que je voudrais qu'ils soient. Je participe depuis suffisamment longtemps aux luttes politiques pour ne pas avoir la naïveté de croire que la cause que l'on défend s'identifie toujours avec la vérité. Il n'existe pas de mouvement social sans contradictions, sans ambiguïtés et donc sans médiations déformantes. Mais il y a des moments où le besoin de vérité redevient un besoin vital, où les pratiques routinières et les idéologies caduques limitent la vue et masquent le chemin, où il faut déchirer le lourd tissu qui nous a peu à peu recouverts pour voir où l'on est et jusqu'où l'on peut aller» (p. 12).

Les syndicalismes observés et comparés sont ceux des pays les plus industrialisés du monde: Grande-Bretagne, République fédérale allemande, Suède, Italie, France, EtatsUnis et Japon. Leur production globale dépasse celle de toutes les autres nations réunies et la concentration des travailleurs salariés y est plus forte que partout ailleurs: c'est bien là, comme le note l'auteur, que l'on peut parler du mouvement ouvrier.

Le syndicalisme de chaque pays fait l'objet d'un chapitre particulier et l'auteur consacre un long chapitre final à répondre à la question "Quel avenir pour le mouvement ouvrier?»

Tout au long de son ouvrage, l'auteur procède de la même façon. Il présente une vue générale de l'histoire de chacun des syndicalismes avec le contexte dans lequel il est né et a évolué. Il expose les aspirations, les revendications, les méthodes d'action des syndicats, leur organisation interne et les résultats qu'ils ont obtenus. Il met aussi en regard les différentes expériences en faisant ressortir les similitudes et les contradictions et trace des perspectives d'évolution. Mais, il avertit le lecteur: "Il s'agit bien entendu de tendances actuellement décelables. Bien des élé ments peuvent les modifier. Mon propos n'est pas de me livrer à un quelconque exercice de futurologie. J'entends seulement situer et dater cette étude».

Parmi les constatations que l'on peut faire, il est des constantes qui se dégagent partout, quels que soient les pays, quels que soient les syndicalismes et quelles que soient les idéologies qui à un moment donné les ont fécondées.

Sans aucun doute, les syndicats ont contribué à améliorer grandement la condition des travailleurs. Mais, en pays capitalistes comme en pays "socialistes», nulle part la classe des travailleurs que représentent les syndicats n'a cessé de vendre sa force de travail en échange d'un salaire; nulle part, la di- 
vision du travail telle qu'amenée par le progrès de l'industrialisation n'a été remis en cause: il y a toujours une distinction entre la direction des entreprises et le personnel d'exécution. Faut-il alors admettre que les ouvriers n'ont pas vraiment vocation de devenir une classe dominante? Le syndicalisme a sa place et son rôle à côté des autres pouvoirs: direction des entreprises, partis politiques, gouvernements. Mais «revendiquer, contrôler et gérer sont des fonctions qui ne sauraient se confondre, même si elles se révèlent complémentaires» (p. 232).

L'auteur est amené à présenter des considérations très judicieuses sur la nature, l'organisation et le rôle du syndicalisme, ses contradictions internes inévitables, les rapports qu'il entretient avec les partis politiques et les gouvernements ainsi que l'influence des idéologies glorifiantes ou mystifiantes.

Dans les perspectives d'avenir, pour rester fidèle à sa mission toujours nécessaire, le syndicalisme devra ajuster son action au contexte nouveau dans lequel il se trouve et tenir compte de certains problèmes dont l'importance ne cessera de croître: la composition de la main-d'oeuvre salariée résultant de la télématique; la répercussion des mutations technologiques sur la gestion des unités économiques avec la signification des projets de cogestion et d'auto-gestion; l'activité des multinationales et l'industrialisation du tiersmonde.

Tous ne seront pas d'accord avec l'un ou l'autre des jugements que porte l'auteur. Cependant, on ne pourra pas lui reprocher de ne pas avoir mis le doigt sur des questions essentielles ni de ne pas avoir soulevé une multitude de questions pertinentes souvent occultées par manque de vision globale du syndicalisme dans le temps et dans l'espace. L'auteur n'a pas réponse à tout. Son ouvrage apporte sans doute certaines démystifications, mais il contribuera à une meilleure compréhension d'une institution qui s'impose au-delà des régimes économiques et politiques aussi longtemps qu'il y aura des travailleurs salariés.

Université Laval
Relations Industrielles ( $2^{\mathrm{e}}$ édition), par Dimitri Weiss, Paris, Coll. «Administration des Entreprises», Éditions Sirey, 1980, $257 \mathrm{pp}$.

On doit rendre à Dimitri Weiss cet hommage: il est, de nos jours, l'un des rares auteurs de langue française, en relations industrielles, à contribuer d'une façon soutenue à une meilleure compréhension réciproque des systèmes de relations industrielles en Amérique du Nord d'une part et en Europe occidentale, d'autre part. Tout en laissant au Professeur Weiss la responsabilité de ses thèses, il faut souligner que sa remarquable contribution, dans le domaine des relations industrielles, ne peut qu'amener des échanges de connaissances - mutuellement enrichissants entre tous ceux et toutes celles qu'intéressent les questions du travail dans nos pays démocratiques. S'il fallait formuler une observation qui s'appliquerait plus particulièrement au livre qui est commenté ici, je dirais que de par son orientation même, il fait mieux comprendre l'universalité de certains problèmes qui se posent sur les lieux du travail, quel que soit leur contexte national.

Ce livre constitue la deuxième édition de l'ouvrage ayant le même titre et que l'auteur avait publié en 1973 aux Éditions Sirey, également (Voir la revue Relations Industrielles, Vol. 28, No 3, pp. 664-665). Il ne s'agit toutefois pas d'un livre simplement mis-à-jour ou remanié. Il s'agit d'un ouvrage qui diffère d'une façon marquée de celui qui l'a précédé en ce qu'il met davantage l'accent sur les causes et le contenu des problèmes qui se posent en relations industrielles plutôt que sur la forme institutionnelle qu'ils revêtent ou sur le cadre formel et juridique au sein duquel ils font surface. La brève citation qui suit, extraite du livre, illustre assez bien, pensonsnous, ce que l'on pourrait peut-être appeler le climat du livre: «En s'occupant uniquement du problème du règlement des conflits, concentré uniquement sur les institutions, et en adoptant une démarche descriptive dans l'explication de la façon dont se nouent les rapports entre les partenaires sociaux dans le cadre de règles et d'institutions données, et ce au détriment de l'étude des relations interper- 